\title{
Kinetic Insights into Cyanosilylation of Aldehydes Catalyzed by a Covalently Bridged Dinuclear (Salen)titanium Complex
}

\author{
Zhipeng Zhang, ${ }^{*[a, b]}$ Zheng Wang, ${ }^{[a]}$ and Kuiling Ding ${ }^{*[a, c]}$
}
[a] Dr. Z. Zhang, Dr. Z. Wang, Prof. Dr. K. Ding
State Key Laboratory of Organometallic Chemistry, Center for Excellence in Molecular Synthesis, Shanghai Institute of Organic Chemistry, Chinese Academy of Sciences, 345 Lingling Road, Shanghai 200032, China
E-mail: kding@mail.sioc.ac.cn
[b] Dr. Z. Zhang
School of Chemistry and Molecular Engineering, Frontiers Science Center for Materiobiology and Dynamic Chemistry, East China University of Science \& Technology, 130 Meilong Road, Shanghai, 200237, China
E-mail: zhipengzhang@ecust.edu.cn
[c] Prof. Dr. K. Ding
Frontiers Science Center for Transformative Molecules, School of Chemistry and Chemical Engineering, Shanghai Jiao Tong University, 800 Dongchuan Road, Shanghai 200240, China

Supporting information for this article is given via a link at the end of the document.

\begin{abstract}
Enantioselective addition of trimethylsilyl cyanide (TMSCN) to aldehydes is one of the most extensively studied organic reactions in asymmetric catalysis. Herein, we report our intensive kinetic investigation on the asymmetric addition of TMSCN to benzaldehyde, catalyzed by a covalently bridged dinuclear (salen)titanium complex 2, which has been one of the most efficient artificial chiral catalysts reported so far for this reaction. It was found that the method of initial rates for kinetic investigation is not appropriate in this case because of the presence of a significant incubation period in the catalysis, while the method of progress rates proved to be more reliable and efficient for judging the kinetic orders of this catalytic system. The kinetic results revealed that the reaction follows first order with respect to the catalyst and is nearly independent of concentrations of both benzaldehyde and TMSCN. A detailed catalytic mechanism for cyanosilylation of benzaldehyde in the presence of 2 was proposed, wherein the key active dinuclear species works in a cooperative manner for dual activation of both reactants.
\end{abstract}

\section{Introduction}

Catalytic asymmetric addition of cyanide to aldehydes and ketones produces enantioenriched cyanohydrins, one type of versatile chiral intermediates in synthesis. ${ }^{[1]}$ During the past decades, a large number of catalysts, including enzymes, ${ }^{[2]}$ Lewis acidic metal-based catalysts, ${ }^{[3]}$ organocatalysts ${ }^{[4]}$ and metalorganic frameworks ${ }^{[5]}$ have been developed for these transformations. ${ }^{[1-5]}$ One of the important breakthroughs in this area is the use of chiral catalyst of dimeric dinuclear (salen)titanium complex 1 (Scheme 1), developed by Belokon', North and co-workers, which demonstrated very high efficiency in the catalytic addition of TMSCN to aldehydes with moderate to high enantioselectivity $(50-92 \%$ ee) at $0.1 \mathrm{~mol} \%$ catalyst loading. ${ }^{[6]}$ Kinetic investigation disclosed that reaction orders are in the range of 1.3-1.8 for several homologous dimeric dinuclear (salen)titanium complexes, suggesting that two metal centers were possibly involved in the catalysis. ${ }^{[7]}$

Inspired by this study, a dinuclear (salen)titanium complex 2 with cis-5-norbornene-endo-2,3-dicarboxylate as a covalent bridge between the two salen-Ti units has been developed, ${ }^{[8]}$ which can effectively prevent the problematic dissociation of the catalytically active dimer $\mathbf{1}$ into inactive salen- $\mathrm{Ti}=\mathrm{O}$ monomers, ${ }^{[7]}$ thereby leading to a dramatic increase of catalytic efficiency. In fact, catalyst 2 demonstrated extremely high efficiency in the asymmetric cyanation of aromatic aldehydes, affording the adducts with ee values up to $97 \%$ even at catalyst loading as low as $0.0005 \mathrm{~mol} \%$ (turnover numbers up to 172000 ). ${ }^{[8]}$ Preliminary mechanistic study suggested a possible intramolecularly cooperative catalysis mechanism, whereas the kinetic behavior of the reaction especially the order in catalyst remained to be explored. Experimental kinetic studies are essential in mechanistic study of a reaction, since the rate dependence on the concentrations of the catalysts and reactants is valuable to understanding the catalysis at the molecular-level. Herein, we will report our detailed kinetic study of cyanosilylation of benzaldehyde $\mathbf{3}$ catalyzed by dinuclear (salen)titanium complex $\mathbf{2}$, which has provided a strong support for a cooperative bimetallic catalysis pathway.
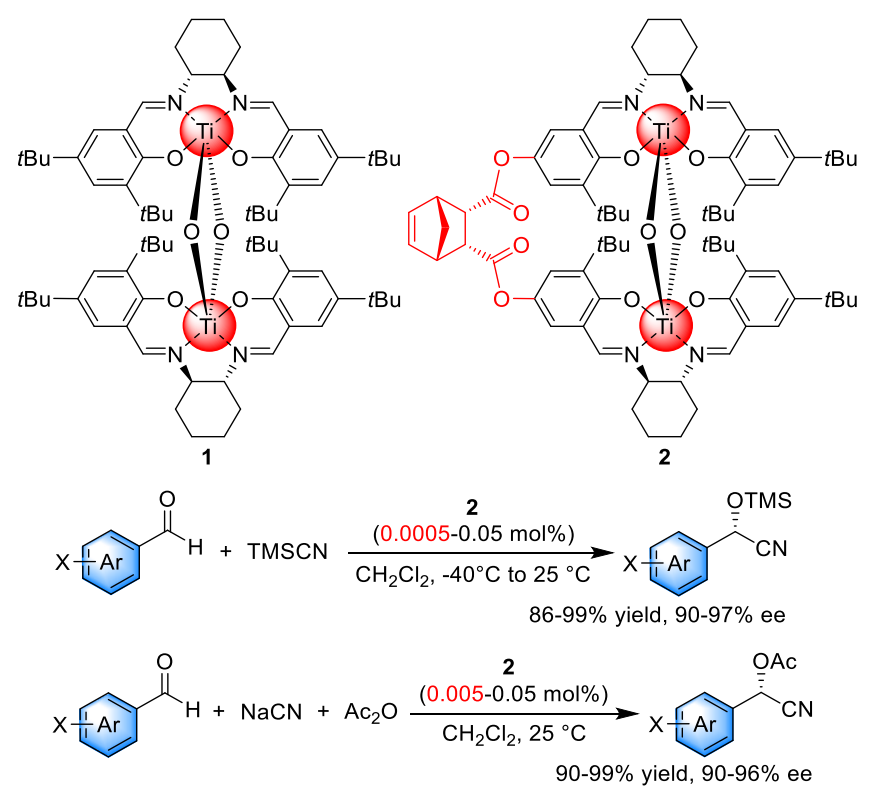

Scheme 1. Dimeric dinuclear (salen)titanium complex 1 reported by Belokon' and North, and covalently bridged dinuclear (salen)titanium complex $\mathbf{2}$ for catalytic asymmetric cyanation of aldehydes in this group.

\section{Results and Discussion}


Attempt to deduce the order in catalyst 2 using the method of initial rates. For the reaction between benzaldehyde 3 and TMSCN 4 with dinuclear (salen)titanium complex 2 as a catalyst, kinetic studies were carried out by monitoring of reaction progress with in situ FT-IR (see Supporting Information for details). At the beginning of the study, several reactions were run at different catalyst loadings ranging from 0.01 to $0.02 \mathrm{~mol} \%$ under otherwise identical conditions, and the initial rates were measured to determine the order in catalyst 2 . The initial rates were thus obtained from the data between $0-5 \%$ and $0-10 \%$ conversion of benzaldehyde 3, respectively, in the reaction profiles established by plotting concentration of benzaldehyde [3] vs time (Figure 1a, 1b). However, the plot of log (rate) vs log [2] (Figure 2a) displayed no sign of acceptable linear relationship, suggesting that the classic method of initial rates does not work well for kinetic studies in this case, presumably caused by the existence of an incubation period for activation of the pre-catalyst 2.

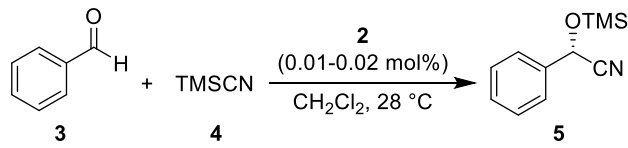

a)

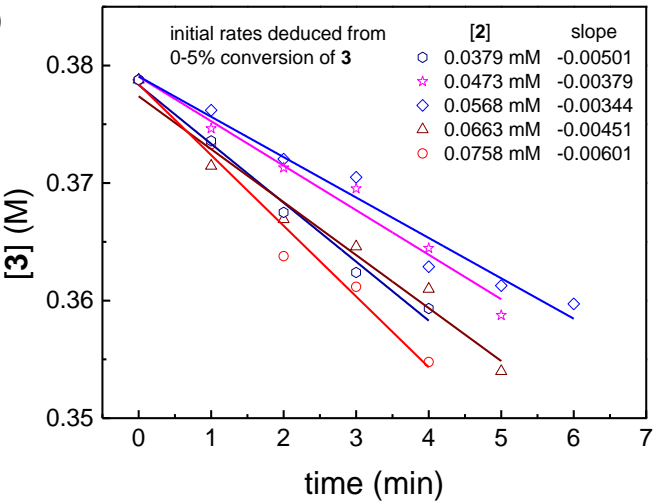

b)

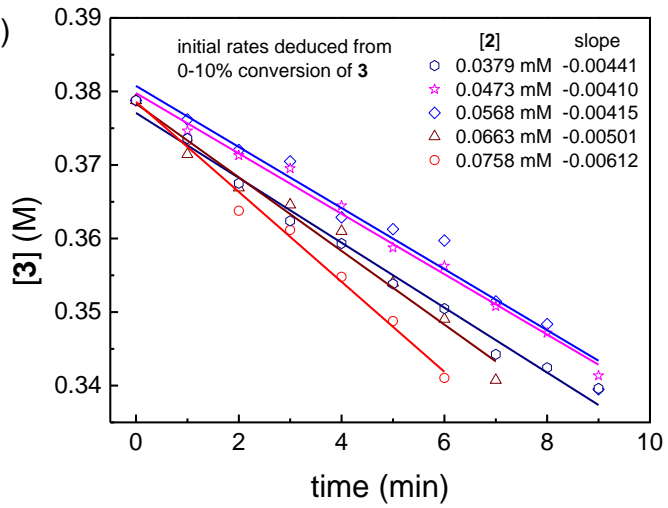

Figure 1. Deduction of the initial rates from the data between (a) $0-5 \%$ and (b) $0-10 \%$ conversion of benzaldehyde 3 . Reagents and conditions: catalyst 2 (0.01-0.02 mol\%, 0.0379-0.0758 mM), initial concentration of benzaldehyde: $[3]_{0}=0.38 \mathrm{M}$, initial concentration of TMSCN: $[4]_{0}=0.42 \mathrm{M}, \mathrm{CH}_{2} \mathrm{Cl}_{2}(15 \mathrm{~mL})$.

A closer examination of the profiles for the entire reaction course (Figure 2b, and Figure S1 in Supporting Information) revealed that there was a tangible rate-accelerating stage in the initial phase of the reactions, especially when the conversion of $\mathbf{3}$ was lower than $20 \%$ (data for Stage 1 were encircled in the red rectangle in Figure 2b). This is usually taken as a clear sign of incubation period, which is the time required for transformation of a pre-catalyst into the active species and for the catalytic reaction to reach the steady state. Incubation period is very common in catalytic reactions, since any catalytic reaction that starts with a precatalyst rather than the real catalytically active species should possess an incubation period in principle, no matter it is short or long, detectable or not. ${ }^{[9]}$ While the classic method of initial rates is widely used to deduce kinetic orders using the kinetic data from the initial stage of a reaction, there is a high risk of encountering an incubation period. If a kinetic study of the catalytic reaction is based on the method of initial rates, the kinetic data obtained from the incubation period will inevitably lead to inaccurate or even incorrect conclusions. Nowadays, various highly accurate in situ spectroscopic techniques have been available for routine monitoring the entire courses of many catalytic reactions, and thus remarkably facilitating the acquisition of high-quality data for kinetic analysis. In this case, the main progress of the reaction should occur at Stage 2 where the conversion of benzaldehyde 3 lies between $20-80 \%$ (Figure $2 b$ ), and data corresponding to this stage are more valuable for the kinetic analysis since the information should reflect the behavior of the reaction in its steady state. The data in Stage 3 (Figure $2 \mathrm{~b}$ ) where the conversion of 3 is higher than $80 \%$ were discarded in pursuing a rate law that reflects steady-state catalysis, as a catalytic reaction usually deviates from the steady state (if it used to reach) near the end of the reaction due to low concentrations of reactants, high concentration of products and/or possible deactivation of catalyst.

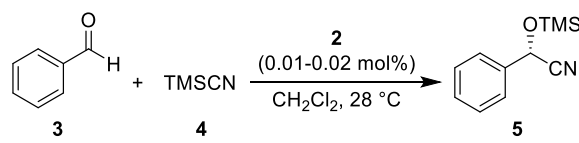

a)

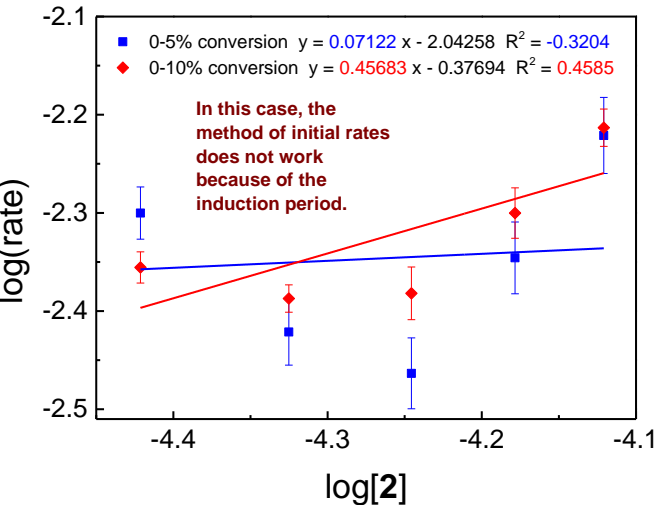

b)

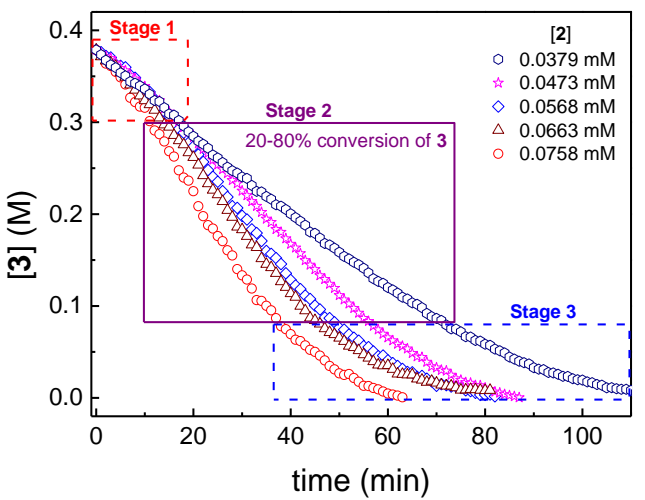


Figure 2. (a) Attempt to deduce the order in catalyst 2 by using the method of initial rates (The plots and the error bars in the Figures of the text were generated with program Origin from OriginLab Corp., Northampton, MA); (b) Different stages in the [3] vs time reaction profiles. Reagents and conditions: catalyst 2 (0.01-0.02 mol\%, 0.0379-0.0758 mM), initial concentration of aldehyde: $[3]_{0}=0.38 \mathrm{M}$, initial concentration of TMSCN: [4] $]_{0}=0.42 \mathrm{M}, \mathrm{CH}_{2} \mathrm{Cl}_{2}$ (15 mL).

Determination of the order in catalyst 2 using the method of progress rates. In the previous kinetic study of disulfonimidecatalyzed cyanosilylation of aldehydes, a novel protocol for kinetic analysis of catalytic reactions, i.e., the method of progress rates, has been developed. The protocol was demonstrated to be very efficient in deducing the orders from a series of kinetic data obtained using in situ measurements. ${ }^{[10]}$ This method allows to study the natural state of the reaction under real conditions where reactant concentrations change at the same time and makes full use of the highly qualified kinetic data mainly obtained from the steady state catalytic cycles of the entire reaction progress. The method of progress rates was employed here for kinetic analysis of the titled reaction, using the data collected from in situ FT-IR studies (for details, see Supporting Information). To this end, the reaction profiles ([3] vs time, Figure $2 b$ ) for the catalysis with different concentrations of $\mathbf{2}$ were first converted to the plots of rates against [3], which delineate clearly the rate variance with the continuously changing concentrations of benzaldehyde over the reaction course (Figure 3a). As shown in each of these profiles, the curves within the region of Stage 2 depicts a well-behaved reaction kinetics, with a smooth curvature much better defined than those in either Stage 1 or 3 . The incubation period are clearly discernible from the profiles, and the initial rates (Stage 1) exhibits an irregular pattern, possibly as a result of the partial activation of the pre-catalyst at the initial stage of the reaction.

Subsequently, as a step towards the derivation of the relationship between rate and [3], the data in each of the profiles in the region of Stage 2 (Figure 3a) were fitted with a distinct highorder polynomial function (Figure $3 b$, blue lines). From the resulting functions, the progress rates (instantaneous reaction rates at a certain time point in the progress of the reaction) for the reactions under the specified catalyst loading can be calculated at a given concentration of aldehyde 3 . This manipulation allows to compare the progress rates at the same phase (for example, the phase indicated by the purple dashed lines in Figure 3a,3b) of the reaction course where the instantaneous concentrations of the reactants such as [3] or [4] from different reactions are the same. Then, a series of concentrations of 3 in Stage 2 (0.08-0.30 $\mathrm{M})$ at a fixed interval (here $0.02 \mathrm{M}$ ), were used to calculate the progress rates, thus affording the data set of \{rate, [2]\} that can be used to derive the reaction kinetics with respect to the catalyst 2 (Figure 3b). Indeed, linear regression of the plot of log (rate) vs log [2] at each [3] (Figure 3c, exemplified with the case of [3] = $0.22 \mathrm{M}$ ) gave a linear function (Figure 3c), whose slope corresponds to the empirical kinetic order of [2] at this specified concentration of 3. Finally, the calculated kinetic order of [2] was plotted against [3] (Figure 3d), giving a profile that reflects the variance in the empirical order of the catalyst as a function of changing substrate concentrations. As shown in Figure 3d, for [3] in the range of $0.08-0.30 \mathrm{M}$ the empirical order in catalyst 2 is 0.96 \pm 0.09 (the calculation of the standard errors was performed with program Origin from OriginLab Corp., Northampton, MA), indicating that the reaction rate is approximately first order with respect to the concentration of the catalyst.

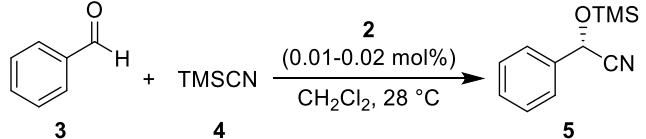

a)

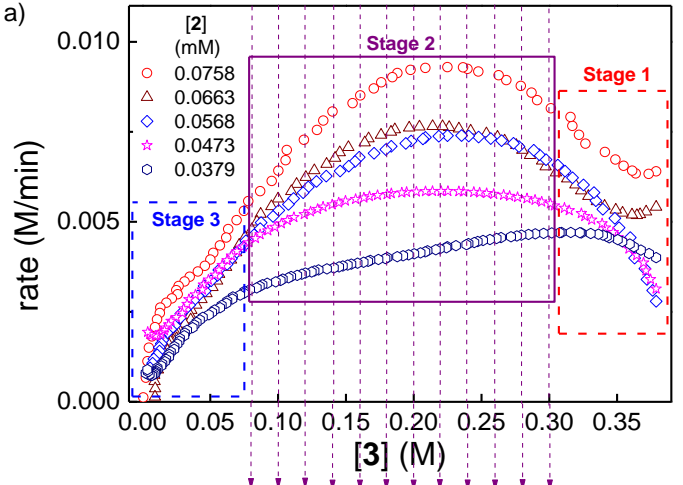

b)
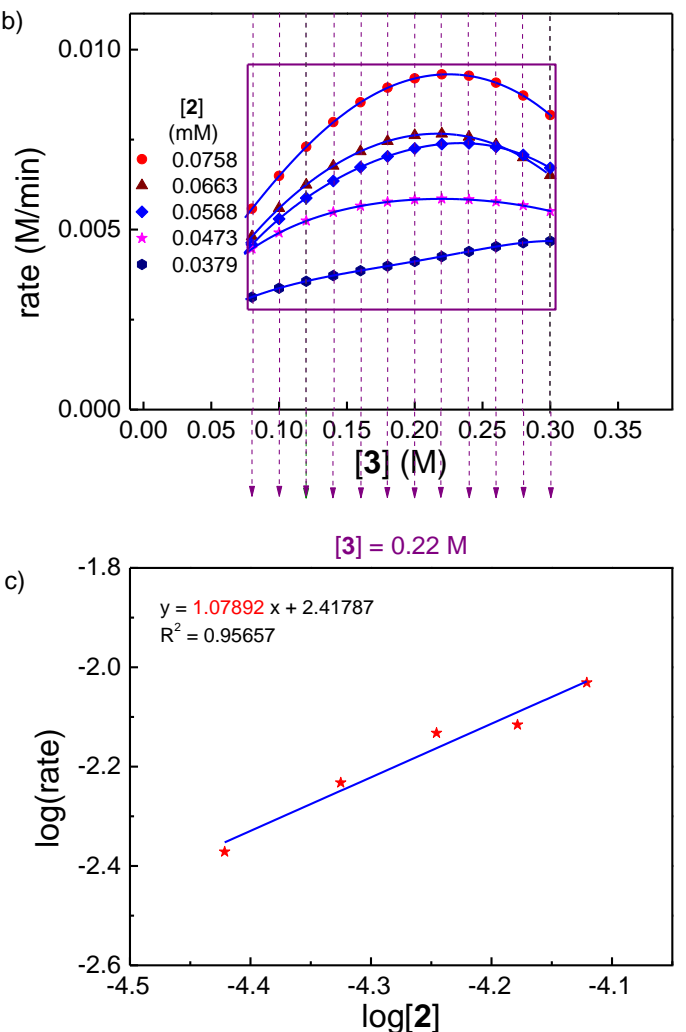

d)

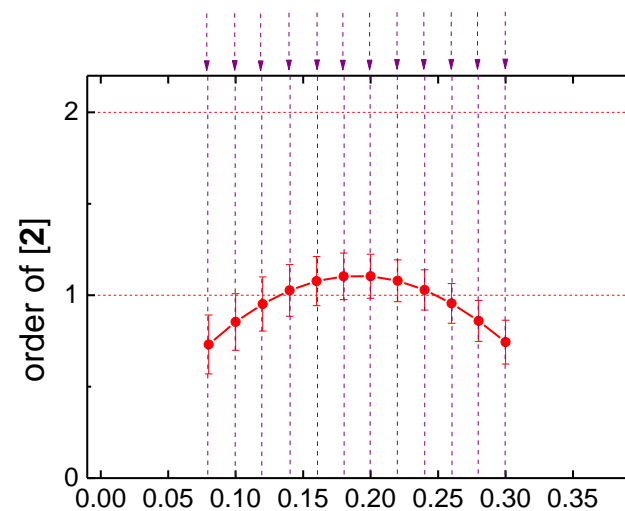

[3] (M) 
Figure 3. Determination of the order of catalyst 2 by using the method of progress rates: (a) Rate vs [3] profiles with various concentration of 2 (catalyst loadings); (b) Fitted high-order polynomial functions (shown as blue lines) and the resulting data sets of \{rate, [2]\} (c) Double logarithmic plot and linear regression of rate vs [2] when [3] $=0.22 \mathrm{M}$ (the deduced order of [2] corresponds to the slope: 1.07892); (d) The profile of (order of [2]) vs [3] in the selected range of [3] $(0.08-0.30 \mathrm{M})$. Reagents and conditions: catalyst $2(0.01-0.02 \mathrm{~mol} \%$, 0.0379-0.0758 mM), initial concentration of aldehyde: $[3]_{0}=0.38 \mathrm{M}$, initial concentration of TMSCN: [4] $]_{0}=0.42 \mathrm{M}, \mathrm{CH}_{2} \mathrm{Cl}_{2}(15 \mathrm{~mL})$.

The obtained arch-shaped kinetic order profile in Figure 3d is very interesting, as it contains clues valuable for understanding the intrinsic kinetic behaviors of the reaction. We speculated that at the initial stage of the reaction (low conversion, right side of the arch-shaped curve), the apparent order in catalyst $\mathbf{2}$ is smaller than the average value owing to the incubation period when only partial activation of the pre-catalyst has been achieved, since higher catalyst loading may cause longer incubation period (or may have lower pre-catalyst activation percentage at same conversion of the catalytic reaction). The calculated order of the catalyst increases gradually with the proceeding of the reaction, and reaches its maximum (about 1.1), and the reaction in the region around this time point (when [3] $=0.18-0.20 \mathrm{M}$ ) is proposed to be the closest to the steady state, which is a theoretical situation that may never really achieve for some catalytic reactions. From this critical point on to the end of the reaction, the calculated catalyst order decreases steadily (left side of the arch shaped curve), possibly due to unsaturation of the catalyst (catalyst with higher loading requires higher reactant concentrations to saturate).

Verification of the order in catalyst 2 via linear regression approach for modelling the [3] vs time profiles in the main progress (Stage 2). A closer examination of the profiles for [3] vs time in Stage 2 (Figure 2b) suggested a quasi-linear relationship between [3] and time. Therefore, we decided to use linear regression approach to model the [3] vs time profiles in Stage 2, and use the thus obtained overall approximate progress rates to deduce the order in catalyst $\mathbf{2}$, so as to further verify the results obtained in the previous section. As shown in Figure 4a, there does exist a fairly good linear relationship between [3] and time. Thus, the obtain progress rates (absolute value of the slopes) were used to deduce the order in catalyst 2 via double logarithmic plot and linear regression of rate vs [2] (Figure 4b). In this way, the empirical order in catalyst 2 was determined to be $1.05 \pm 0.17$, which is in good agreement with the previously obtained result $(0.96 \pm 0.09)$, thus confirming first order in catalyst 2 . This result indicated that only one molecule of dinuclear (salen)titanium complex $\mathbf{2}$ is involved in the rate-determining step, and supported the catalytically active dinuclear species and cooperative dual activation model proposed by Belokon' and North. ${ }^{[6,7]}$ The dicarboxylate bridge in dinuclear (salen)titanium complex 2 effectively prevent the two salen-Ti units from dissociation during catalysis. This explains why complex $\mathbf{2}$ is such a highly efficient catalyst in cyanosilylation of benzaldehyde, which proceeded well even at a catalyst loading of only $0.0005 \mathrm{~mol} \%$.

Determination of the kinetic orders in benzaldehyde 3 and TMSCN 4. There exists a fairly good linear relationship between [3] and time in stage 2 (Figure $2 \mathrm{~b}$ and $4 \mathrm{a}$ ), implying that the progress rates are nearly constant, i.e. the reaction displays nearly zero order kinetics in a large part of the reaction progress.
That is to say, the orders both in benzaldehyde 3 and in TMSCN 4 should be very close to zero, thus leading to a nearly constant rate under a fixed catalyst loading. To verify this rationale, further experiments were carried out under identical conditions, by varying only the initial concentrations of benzaldehyde 3 or TMSCN 4. The resulting kinetic plots were shown in Figure 5 and 6 , respectively. Two reaction profiles of [4] vs time corresponding to initial concentrations of $\left\{[4]_{0}=0.59 \mathrm{M},[3]_{0}=0.38 \mathrm{M}\right\}$ and $\left\{[4]_{0}\right.$ $\left.=0.59 \mathrm{M},[3]_{0}=0.55 \mathrm{M}\right\}$ were obtained, and the data sets between [4] $=0.28-0.46 \mathrm{M}$ (Figure 5a) were modelled with linear regression approach. The resultant overall approximate progress rates (absolute value of the slopes) were used to calculate the order in benzaldehyde 3 , via double logarithmic plot and linear regression of rate vs [3] (Figure $5 b$ ). In this way, the average value of the theoretical order in 3 was determined to be $0.14 \pm 0.03$ which is in good agreement with our assumption (nearly zero order).

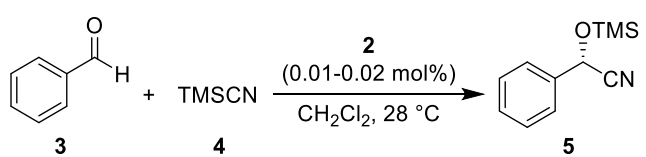

a)
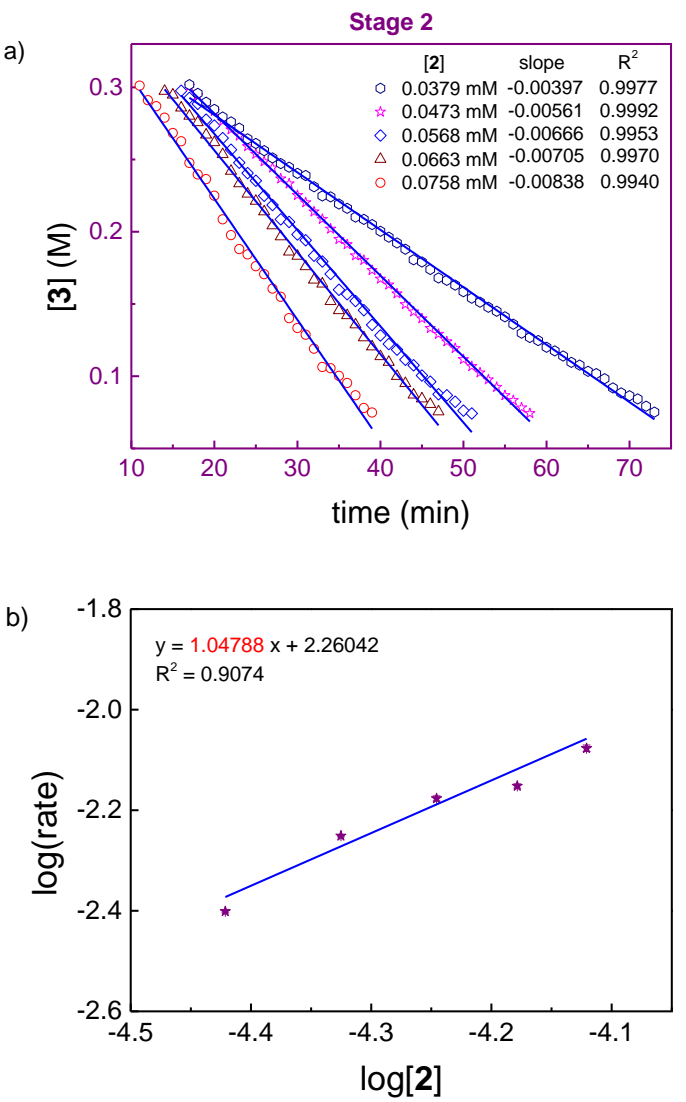

Figure 4. Determination of the order of catalyst 2 via linear regression approach for modelling the [3] vs time profiles in the main progress (Stage 2): (a) Deduction of the progress rates via linear regression of [3] vs time profiles; (b) Double logarithmic plot and linear regression of rate vs [2] (the deduced order of [2] corresponds to the slope: 1.0479).

In a similar way (Figure 6), the average value of the empirical order in TMSCN 4 was determined to be $0.08 \pm 0.01$, which is also in good agreement with our speculation (nearly zero order). 
Taking all these results together, the overall empirical rate law (power-law form) of the reaction, which reflects mechanistic events at molecular-level, can be expressed in Equation 1. It is worth to note that this empirical rate equation approximately reflects the kinetic behavior in the main progress of the reaction (around $20-80 \%$ conversion of 3 ) while may not be applicable to the initial or final phase of the reaction.

$$
\text { rate }=k \cdot[2]^{1.0} \cdot[3]^{0.14} \cdot[4]^{0.08} \quad(\text { Equation 1) }
$$

a)

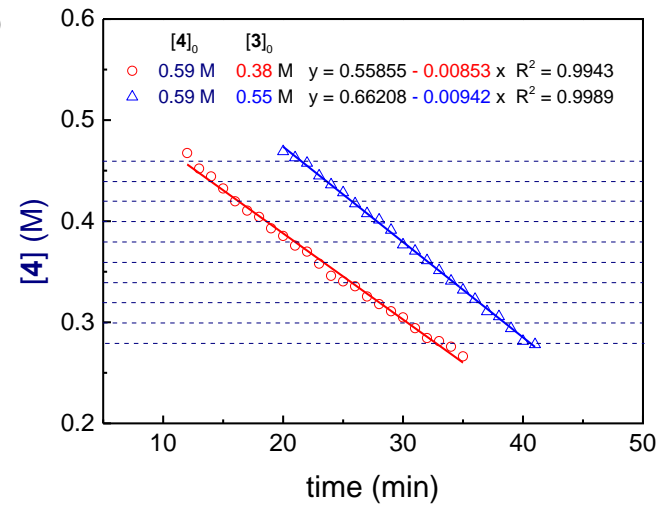

b)

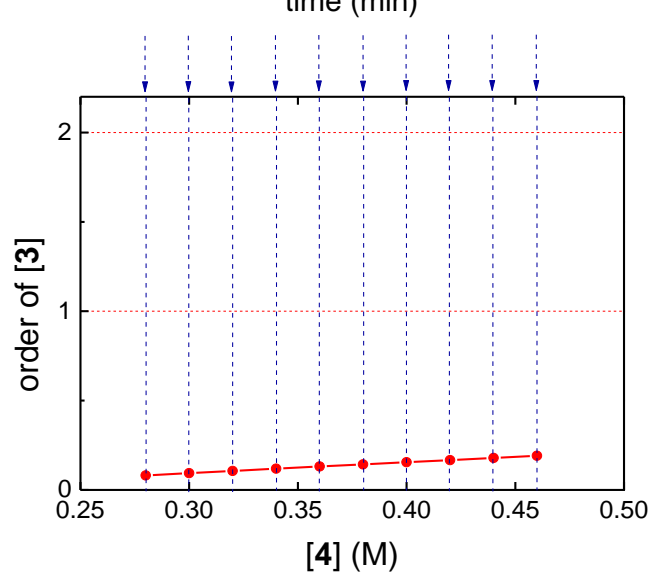

Figure 5. Determination of the order of benzaldehyde 3 via linear regression of [4] vs time profiles in the main progress. (a) Profiles of [4] vs time with same initial concentration of TMSCN: $[4]_{0}=0.59 \mathrm{M}$ and different initial concentration of benzaldehyde: [3 $]_{0}=0.38,0.55 \mathrm{M}$; (b) Profile of (order of [3]) vs [4]. Reagents and conditions: catalyst $2(1.66 \mathrm{mg}, 0.0758 \mathrm{mM}), 28^{\circ} \mathrm{C}, \mathrm{CH}_{2} \mathrm{Cl}_{2}(15 \mathrm{~mL})$.

Determination of the apparent activation energy, activation enthalpy and entropy. The temperature dependence of the reaction rates was studied in the temperature range of 296.15$311.15 \mathrm{~K}$ under otherwise identical conditions (Figure 7a). The apparent activation energy of the reaction was deduced to be 34.5 $\pm 1.5 \mathrm{~kJ} \cdot \mathrm{mol}^{-1}\left(8.24 \pm 0.36 \mathrm{kcal} \cdot \mathrm{mol}^{-1}\right)$ according to the Arrhenius equation by plotting In $k$ vs $1 / T$ (Figure $7 \mathrm{~b}$ ). The activation enthalpy $\Delta H^{\ddagger}$ was deduced to be $32.0 \pm 1.5 \mathrm{~kJ} \cdot \mathrm{mol}^{-1}(7.64 \pm 0.36 \mathrm{kcal} \cdot \mathrm{mol}$ $\left.{ }^{1}\right)$ and the activation entropy $\Delta S^{\ddagger}$ was deduced to be $-95.7 \pm 5.0$ $\mathrm{J} \cdot \mathrm{mol}^{-1} \cdot \mathrm{K}^{-1}\left(-22.9 \pm 1.2 \mathrm{cal} \cdot \mathrm{mol}^{-1} \cdot \mathrm{K}^{-1}\right)$ according to the Eyring equation by plotting $\ln (k / T)$ vs $1 / T$. The Gibbs energy of activation $\Delta G^{\ddagger}$ was calculated to be $58.1 \pm 2.9 \mathrm{~kJ} \cdot \mathrm{mol}^{-1}(13.9 \pm 0.68 \mathrm{kcal} \cdot \mathrm{mol}$ $\left.{ }^{1}\right)$ at $273.15 \mathrm{~K}$ (Supporting Information Figure S13).

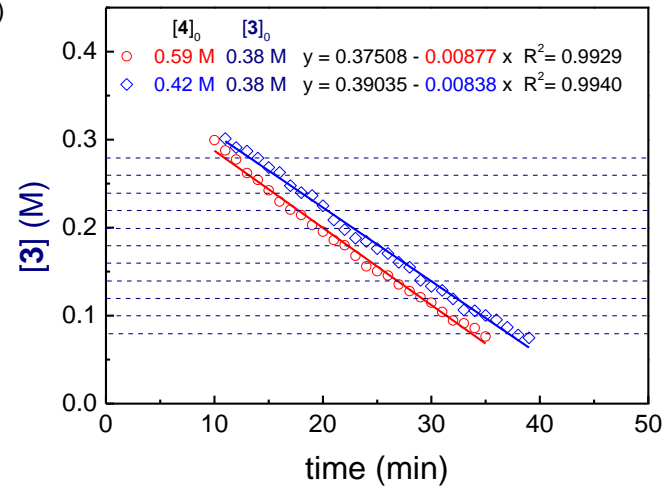

b)

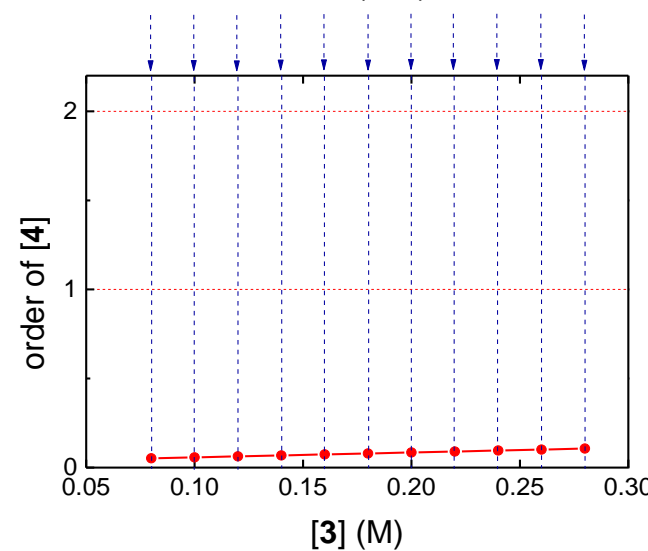

Figure 6. Determination of the order of TMSCN 4 via linear regression of [3] vs time profiles in the main progress. (a) Profiles of [3] vs time with same initial concentration benzaldehyde: $[3]_{0}=0.38 \mathrm{M}$ and different initial concentration of TMSCN: [4] $]_{0}=0.42,0.59 \mathrm{M}$; (b) Profile of (order of [4]) vs [3]. Reagents and conditions: catalyst $2(1.66 \mathrm{mg}, 0.0758 \mathrm{mM}), 28^{\circ} \mathrm{C}, \mathrm{CH}_{2} \mathrm{Cl}_{2}(15 \mathrm{~mL})$

The proposed mechanism. Based on the kinetic investigation results described above, as well as the high similarity between 2 and dimeric dinuclear (salen)titanium complex $\mathbf{1}$, and the catalytic cycle and transition state proposed by Belokon' and North for complex $1,{ }^{[6,7]}$ a catalytic cycle for cyanosilylation of benzaldehyde catalyzed by the covalently bridged dinuclear (salen)titanium complex 2 was outlined as shown in Scheme 2. Nearly zero order both in $\mathbf{3}$ and $\mathbf{4}$ indicates none of them directly participates in the rate-determining step as a free molecule. Possibly a catalyst species associated with both benzaldehyde 3 and the nucleophilic cyanide anion is involved in the rate-determining step, and the intramolecular attack of a Ti-bound $\mathrm{CN}$ on the Ti-activated aldehyde is the turn-over limiting step. Within the catalytic cycle, the transmetallation reaction of TMSCN 4 with the cyanohydrinbound $\mathrm{Ti}$ intermediate to release the cyanosilylation product $\mathbf{5}$ and regenerate the $\mathrm{CN}$-loaded catalytic species should be fast. As shown in Scheme 2, during the incubation period the pre-catalyst complex 2 reacts with two molecules of TMSCN (4), to generate the catalytically active species $\mathbf{A}$ with the release of $(\mathrm{TMS})_{2} \mathrm{O}$ (step 1). Since complex 2 is also possible to present as a polymeric/oligomeric form via $\mathrm{O}-\mathrm{Ti}-\mathrm{O}$ to $\mathrm{Ti}=\mathrm{O}$ rearrangement, ${ }^{[7]} \mathrm{a}$ depolymerization process can't be ruled out during the precatalyst activation period. A undergoes ligand exchange with benzaldehyde $\mathbf{3}$, to afford key bimetallic species B (step 2), in which one titanium acts as Lewis acid to activate aldehyde and the other brings the nucleophilic cyanide in proximity. Such kind 
of dual activation of substrates and precise preorganization of the reaction components allow the subsequent intramolecular transfer of cyanide to the activated aldehyde (step 3) in a very efficient and stereoselective way to afford the titanium bonded cyanohydrin C. Finally, the reaction of titanium bonded cyanohydrin $\mathbf{C}$ with TMSCN releases TMS protected product $\mathbf{5}$ and regenerate intermediate $\mathbf{A}$ for the next cycle. Neither step 2 nor step 4 can be the rate-determining step since the reaction rate is nearly independent of substrate concentrations as disclosed in the kinetic studies. The rate-determining step most probably is the intramolecular nucleophilic attack of cyanide to benzaldehyde in the intermediate $\mathbf{B}$ (step 3), which is supported by the first order kinetic behavior of catalyst $\mathbf{2}$ and the independence of enantioselectivity on employed cyanide sources (both TMSCN and $\mathrm{NaCN}$ afford the cyanation products with $96 \%$ ee), ${ }^{[\mathrm{aa}]}$ as well as the very negative activation entropy $\left(-95.7 \pm 5.0 \mathrm{~J} \cdot \mathrm{mol}^{-1} \cdot \mathrm{K}^{-1}\right)$.

a)

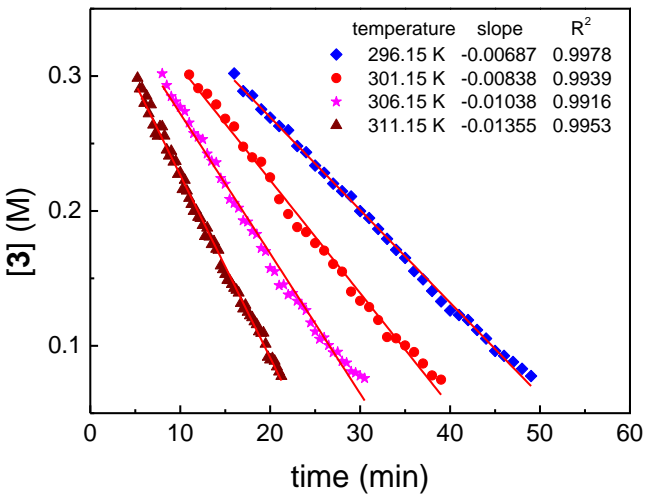

b)

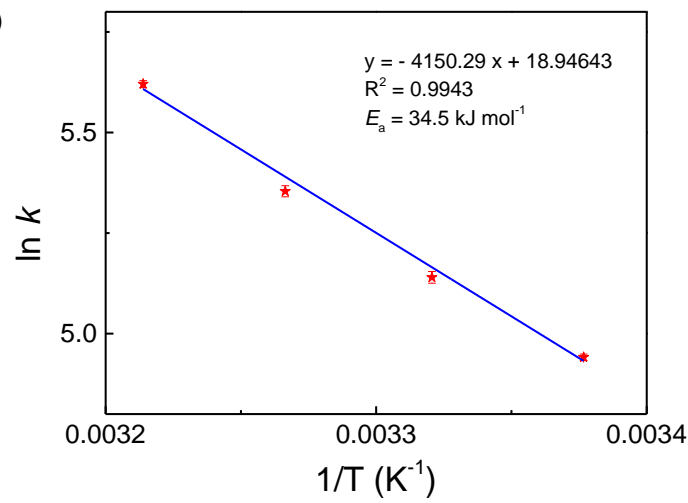

Figure 7. Determination of the apparent activation energy: (a) Profiles of [3] versus time obtained at different temperatures; b) Profile of In $k$ vs $1 / T([3]=0.2$ M). Reagents and conditions: $[3]_{0}=0.38 \mathrm{M},[4]_{0}=0.42 \mathrm{M}, \mathrm{CH}_{2} \mathrm{Cl}_{2}(15 \mathrm{~mL}), 2$ (1.66 mg, $0.02 \mathrm{~mol} \%, 0.0758 \mathrm{mM}$ ), 296.15, 301.15, 306.15, 311.15 K.

\section{Conclusion}

The kinetic behaviors of the cyanosilylation of benzaldehyde catalyzed by the covalently bridged dinuclear (salen)titanium complex have been investigated with in situ FT-IR technique, and the empirical orders in catalyst and reactants for a power-law form of rate equation are deduced through the method of progress rates. It is worth mentioning that the method of initial rates did not work in this case because of the incubation period and particular attention is deserved whenever the method of initial rates is employed to deduce kinetic orders of catalytic reactions that have incubation period. The reaction kinetics can be approximately described as a first order dependence on the dinuclear catalyst $\mathbf{2}$, and nearly zero order on both aldehyde and TMSCN. Such a kinetic behavior is consistent with the proposed mechanistic scenario of a bimetallic synergistic catalysis, wherein an intramolecular nucleophilic attack of cyanide anion to benzaldehyde, both activated within the same catalyst molecule, is likely to be turnover-limiting. The underlying reason for extremely high efficiency of catalyst 2 in cyanosilylation of benzaldehyde can be well explained using this model. It is expected that both the kinetic investigation protocol employed in the present work and the cooperative catalysis model disclosed in this reaction system might stimulate future works on use of progress rate method for kinetic studies of catalytic reactions and the design of new high-performance catalysts in asymmetric reactions. ${ }^{[11]}$
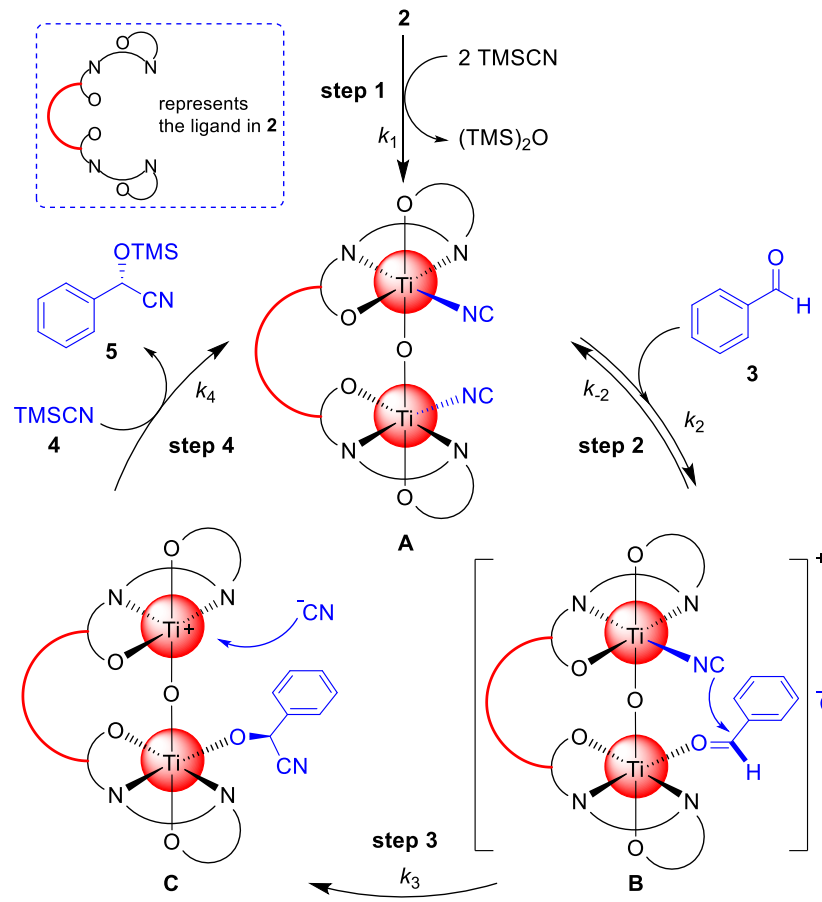

rate-determining step

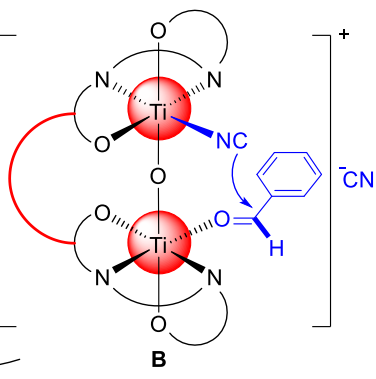

Scheme 2. Proposed catalytic cycle with intramolecular nucleophilic attack of cyanide anion to benzaldehyde as rate-determining step.

\section{Experimental Section}

Caution: TMSCN must be carefully used in a well-ventilated hood due to its high toxicity! Catalyst 2 (1.66 mg, $1.25 \mu \mathrm{mol}, 0.02 \mathrm{~mol} \%, 0.0758 \mathrm{mM}$ ) and $\mathrm{CH}_{2} \mathrm{Cl}_{2}(15 \mathrm{~mL})$ were placed in a pre-dried Schlenk tube equipped with a Teflon-coated magnetic stirring bar and a thermometer under nitrogen. The tube was immersed in a water bath and the temperature was set to be at $28^{\circ} \mathrm{C}$. The probe rod of ReactIR ${ }^{\circledR} 4000$ (Mettler-Toledo) was immersed into the solution and the background spectrum was collected. The scanning started (interval: $1.0 \mathrm{~min})$ and benzaldehyde $3(0.64 \mathrm{~mL}, 6.25$ $\mathrm{mmol}, 0.38 \mathrm{M}$ ) was added via syringe into the solution. The stretching vibration absorption of carbonyl group $\left(1710 \mathrm{~cm}^{-1}\right)$ in 3 was monitored After a few minutes, an aliquot of TMSCN 4 in a syringe $(0.87 \mathrm{~mL}, 6.88$ 
mmol, $0.42 \mathrm{M}$ ) was slowly added to the tube over about 10 seconds and the profile of reaction (IR vs time) was recorded. The reaction was quenched with water $(10 \mu \mathrm{l})$ after most benzaldehyde 3 had been consumed.

\section{Acknowledgements}

This work was supported by National Natural Science Foundation of China (21702059, 21790333), Ministry of Science and Technology of China (2016YFA0202900), the CAS (XDB20000000, QYZDY-SSW-SLH012), Fundamental Research Funds for the Central Universities (222201814014, JKVJ12001010) and Shanghai Pujiang Program (18PJ1402200). We greatly thank Dr. Y.-F. Yang for valuable discussion.

Keywords: Asymmetric catalysis, $\cdot$ Cyanation $•$ Kinetics • Cooperative Catalysis $\cdot$ titanium $\cdot$ Salen

[1] a) X.-P. Zeng, J.-C. Sun, C. Liu, C.-B. Ji, Y.-Y. Peng, Adv. Synth. Catal. 2019, 361, 3281-3305; b) N. Kurono, T. Ohkuma, ACS Catal. 2016, 6 989-1023; c) W. Wang, X. Liu, L. Lin, X. Feng, Eur. J. Org. Chem. 2010, 25, 4751-4769; d) M. North, D. L. Usanov, C. Young, Chem. Rev. 2008 108, 5146-5226; e) E. Wingstrand, S. Lundgren, M. Penhoat, C. Moberg, Pure Appl. Chem. 2006, 78, 409-414; f) J.-M. Brunel, I. P. Holmes, Angew. Chem. 2004, 116, 2810 - 2837; Angew. Chem. Int. Ed. 2004, 43, 2752-2778; g) R. J. H. Gregory, Chem. Rev. 1999, 99, 3649-3682.

[2] a) F. Effenberger, Angew. Chem. 1994, 106, 1609-1619; Angew. Chem. Int. Ed. 1994, 33, 1555-1564; b) F. Effenberger, S. Förster, H. Wajant, Curr. Opin. Biotech. 2000, 11, 532-539; c) H. Gröger, Adv. Synth. Catal. 2001, 343, 547-558; d) E. García-Urdiales, I. Alfonso, V. Gotor, Chem. Rev. 2005, 105, 313-354; e) J. Sukumaran, U. Hanefeld, Chem. Soc. Rev. 2005, 34, 530-542; f) J. Holt, U. Hanefeld, Curr. Org. Synth. 2009 6, 15-37.

[3] a) X.-P. Zeng, Z.-Y. Cao, X. Wang, L. Chen, F. Zhou, F. Zhu, C.-H. Wang, J. Zhou, J. Am. Chem. Soc. 2016, 138, 416-425; b) A. Laurell Nash, R. Hertzberg, Y.-Q. Wen, B. Dahlgren, T. Brinck, C. Moberg, Chem. Eur. J. 2016, 22, 3821-3829; c) Y.-L. Wei, W.-S. Huang, Y.-M. Cui, K.-F. Yang, Z. Xu, L.-W. Xu, RSC Adv. 2015, 5, 3098-3103; d) C. Lv, C.-X. Miao, D. Xu, S. Wang, C. Xia, W. Sun, Catal. Commun. 2012, 27, 138-140; e) M. Uemura, N. Kurono, Y. Sakai, T. Ohkuma, Adv. Synth. Catal. 2012, 354, 2023-2030; f) Y.Q. Wen, W. M. Ren, X. B. Lu, Chin. Chem. Lett. 2011, 22, 1285-1288; g) C. Lv, Q. Cheng, D. Xu, S. Wang, C. Xia, W. Sun, Eur. J. Org. Chem. 2011, 2011, 3407-3411; h) M. North, M. Omedes-Pujol, C. Williamson, Chem. Eur. J. 2010, 16, 11367-11375; i) M. North, P. Villuendas, C. Williamson, Tetrahedron 2010, 66, 1915-1924; j) Y. N. Belokon', W. Clegg, R. W. Harrington, V. I. Maleev, M. North, M. O. Pujol, D. L. Usanov, C. Young, Chem. Eur. J. 2009, 15, 2148-2165; k) N. Kurono, K. Arai, M. Uemura, T. Ohkuma, Angew. Chem. 2008,120, 6745-6748; Angew. Chem. Int. Ed. 2008, 47, 6643-6646; I) B. Zeng, X. Zhou, X. Liu, X. Feng, Tetrahedron, 2007, 63, 5129-5136; m) Y. Liu, X. Liu, J. Xin, X. Feng, Synlett 2006, 7, 1085-1089; n) S. Lundgren, E. Wingstrand, M. Penhoat, C. Moberg, J. Am. Chem. Soc. 2005, 127, 11592-11593; o) M. Hatano, T. Ikeno, T. Miyamoto, K. Ishihara, J. Am. Chem. Soc. 2005, 127, 10776-10777; p) X. Liu, B. Qin, X. Zhou, B. He, X. Feng, J. Am. Chem. Soc. 2005, 127, 12224-12225; q) Y. Hamashima, M. Kanai, M. Shibasaki, J. Am. Chem. Soc. 2000, 122, 7412-7413; r) Y. Hamashima, D. Sawada, M. Kanai, M. Shibasaki, J. Am. Chem. Soc. 1999, 121, 2641-2642.

[4] a) A. Matsumoto, K. Asano, S. Matsubara, Org. Lett. 2019, 21, 2688 2692; b) Y, Kurimoto, T. Nasu, Y. Fujii, K. Asano, S. Matsubara, Org. Lett. 2019, 21, 2156-2160; c) Z. Zhang, H. Y. Bae, J. Guin, C. Rabalakos, M. van Gemmeren, M. Leutzsch, M. Klussmann, B. List, Nat. Commun. 2016, 7, 12478; d) B. A. Provencher, K. J. Bartelson, Y. Liu, B. M.
Foxman, L. Deng, Angew. Chem. 2011, 123, 10753-10757; Angew. Chem. Int. Ed. 2011, 50, 10565-10569; e) S. J. Zuend, E. N. Jacobsen J. Am. Chem. Soc. 2007, 129, 15872-15883; f) D. Peng, H. Zhou, X. Liu, L. Wang, S. Chen, X. Feng, Synlett 2007, 15, 2448-2450; g) B. Qin, X Liu, J. Shi, K. Zheng, H. Zhao, X. Feng, J. Org. Chem. 2007, 72, 23742378; h) S.-K. Tian, L. Deng, Tetrahedron, 2006, 62, 11320-11330; i) D. E. Fuerst, E. N. Jacobsen, J. Am. Chem. Soc. 2005, 127, 8964-8965; j) D. H. Ryu, E. J. Corey, J. Am. Chem. Soc. 2005, 127, 5384-5387; k) Y Wen, X. Huang, J. Huang, Y. Xiong, B. Qin, X. Feng, Synlett 2005, 16, 2445-2448; I) H. Zhou, F.-X. Chen, B. Qin, X. Feng, G. Zhang, Synlett 2004, 6, 1077-1079; m) Y. Li, B. He, X. Feng, G. Zhang, Synlett 2004, 9, 1598-1660; n) D. H. Ryu, E. J. Corey, J. Am. Chem. Soc. 2004, 126 8106-8107; o) S.-K. Tian, R. Hong, L. Deng, J. Am. Chem. Soc. 2003, 125, 9900-9901; p) S.-K. Tian, L. Deng, J. Am. Chem. Soc. 2001, 123, 6195-6196; q) J. Oku, N. Ito, S. Inoue, Makromol. Chem. 1979, 180, 1089-1091.

[5] a) F.-Z. Jin, C.-C. Zhao, H.-C. Ma, G.-J. Chen, Y.-B. Dong, Inorg. Chem. 2019, 58, 9253-9259; b) Z. Li, Y. Liu, X. Kang, Y. Cui, Inorg. Chem. 2018, 57, 9786-9789; c) J. Li, Y. Ren, C. Qi, H. Jiang, Chem. Commun. 2017, 53, 8223-8226.

[6] a) Y. N. Belokon', S. Caveda-Cepas, B. Green, N. S. Ikonnikov, V. N Khrustalev, V. S. Larichev, M. A. Moscalenko, M. North, C. Orizu, V. I. Tararov, M. Tasinazzo, G. I. Timofeeva, L. V. Yashkina, J. Am. Chem. Soc. 1999, 121, 3968-3973. b) V. I. Tararov, D. E. Hibbs, M. B Hursthouse, N. S. Ikonnikov, K. M. A. Malik, M. North, C. Orizu, Y. N Belokon', Chem. Commun. 1998, 387-388.

[7] Y. N. Belokon', B. Green, N. S. Ikonnikov, V. S. Larichev, B. V. Lokshin, M. A. Moscalenko, M. North, C. Orizu, A. S. Peregudov, G. I. Timofeeva, Eur. J. Org. Chem. 2000, 2655-2661.

[8] a) Z. Zhang, Z. Wang, R. Zhang, K. Ding, Angew. Chem. 2010, 122, 6898-6902; Angew. Chem. Int. Ed. 2010, 49, 6746-6750; b) M. North Angew. Chem. 2010, 122, 8255-8257; Angew. Chem. Int. Ed. 2010, 49, 8079-8081.

[9] A. D. McNaught, A. Wilkinson in IUPAC. Compendium of Chemical Terminology, 2nd ed. (the "Gold Book"), Blackwell Scientific, Oxford, 1997.

[10] a) Z. Zhang, M. Klussmann, B. List, Synlett 2020, DOI 10.1055/s-00401707129; b) Z. Zhang, M. Klussmann, B. List, 2020, DOI 10.26434/chemrxiv.12084768.v1.

[11] For a review, see: M. Kanai, in Organic Chemistry: Breakthroughs and Perspectives (Eds.: K. Ding, L.-X. Dai), Wiley-VCH, Weinheim, 2012, pp. 385-401; for the comments by T. Ikariya, T. Ooi, K. Ding, D. Milstein, respectively, see: in Organic Chemistry: Breakthroughs and Perspectives (Eds.: K. Ding, L.-X. Dai), Wiley-VCH, Weinheim, 2012, pp. 401-412. 


\section{Entry for the Table of Contents}

Insert graphic for Table of Contents here.

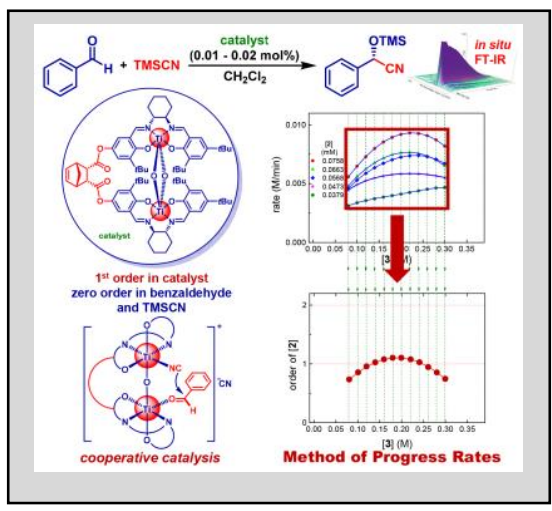

Kinetic investigations using in situ FT-IR technique and the method of progress rates disclosed that cyanosilylation of aldehydes follows first order in catalyst and nearly zero order in aldehyde and TMSCN, supporting an intramolecular cooperative catalysis mechanism. This model has well illustrated the underlying reason for extremely high efficiency of the title catalyst in the reaction. 